\title{
Correction to: Phloretin Inhibits the Human Prostate Cancer Cells through the Generation of Reactive Oxygen Species
}

\author{
Ukjin Kim ${ }^{1}$ (1) $\cdot$ C-Yoon Kim ${ }^{2} \cdot$ Ji Min Lee ${ }^{1} \cdot$ Hanseul Oh ${ }^{1,3} \cdot$ Bokyeong Ryu $^{1} \cdot$ Jin Kim $^{1} \cdot$ Jae-Hak Park ${ }^{1}$
}

Published online: 21 May 2019

(C) Arányi Lajos Foundation 2019

\section{Correction to: Pathology \& Oncology Research} https://doi.org/10.1007/s12253-019-00643-y

The original version of this article unfortunately contained an error in Figs. 1, 5 and 6. The asterisks and bars indicating statistical significance were missing in the figures.

The corrected Figs. 1, 5 and 6 are shown below.

The original article has been corrected.

The online version of the original article can be found at https://doi.org/ 10.1007/s12253-019-00643-y

Jae-Hak Park

pjhak@snu.ac.kr

1 Department of Laboratory Animal Medicine, Research Institute for Veterinary Science, BK21 PLUS Program for Creative Veterinary Science Research, College of Veterinary Medicine, Seoul National University, Seoul 08826, Republic of Korea

2 Department of Stem Cell Biology, School of Medicine, Konkuk University, Seoul 05029, Republic of Korea

3 National Primate Research Center, Korea Research Institute of Bioscience and Biotechnology, CheongjuChungcheongbuk, Republic of Korea 

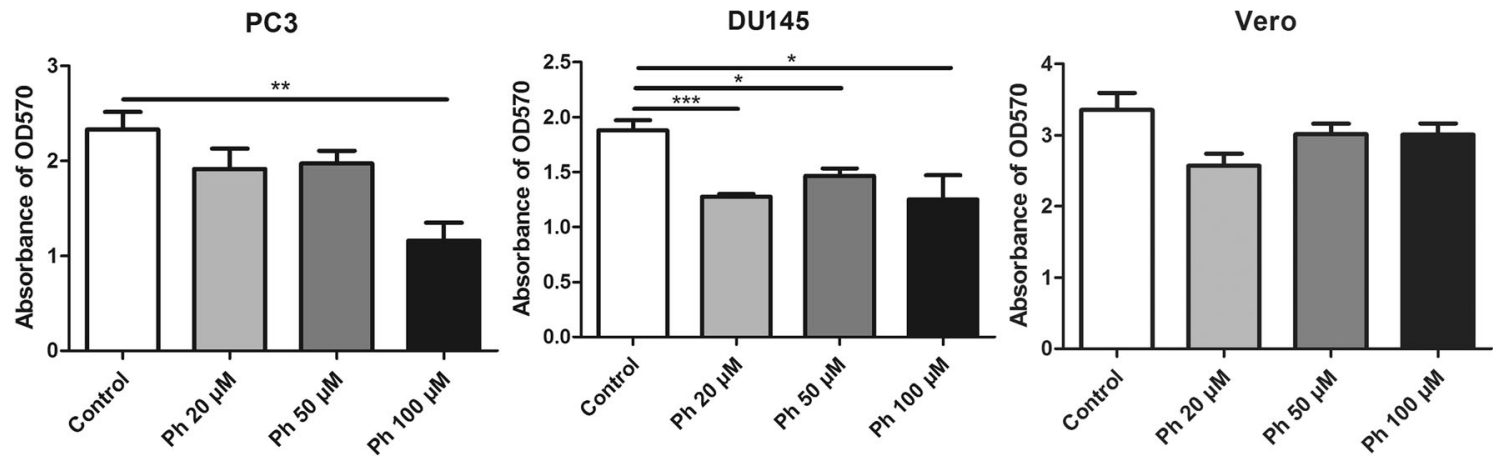

Fig. 1 The effect of phloretin on cell proliferation. The inhibition of proliferation of PC3 and DU145 by phloretin was confirmed by MTT assay. But, phloretin has no effect of normal cell line Vero cells derived from normal African green monkeys. Results are presented as means \pm SEM

PC3

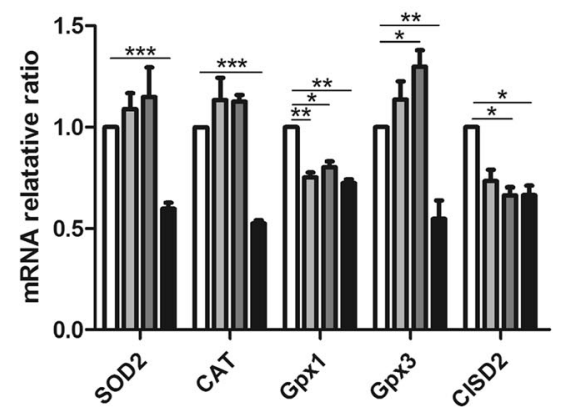

Fig. 5 The effect of phloretin on mRNA expression levels of SOD2, CAT, Gpx1, Gpx3 and CISD2 in PC3 and DU145 cells. Phloretin downregulated the mRNA expression levels of anti-oxidant enzymes in
DU145

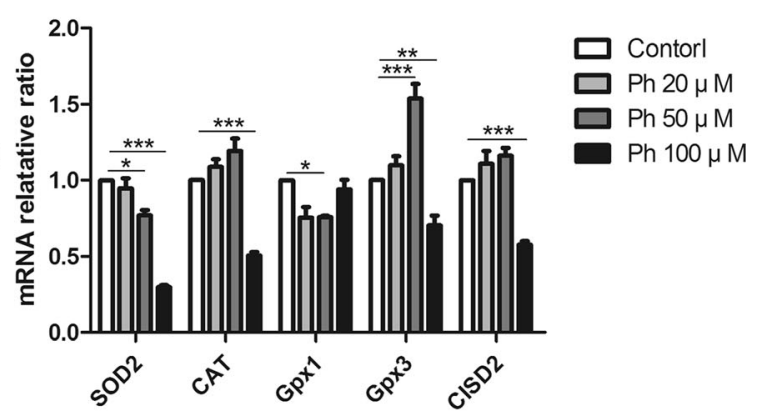

PC3 and DU145 cells. The mRNA expression levels of CISD2 was downregulated in PC3 and DU145 cell. Results are presented as means \pm SEM
PC3

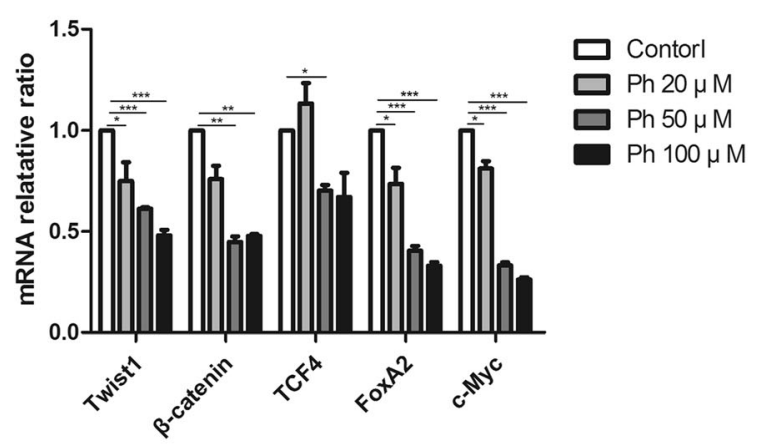

Fig. 6 The effect of phloretin on mRNA expression levels of Twist1, $\beta$ catenin, TCF4, FoxA2, and c-Myc in PC3 and DU145 cells. The mRNA expression levels of Twist1, $\beta$-catenin, TCF 4 and FoxA2 in PC3 and
DU145

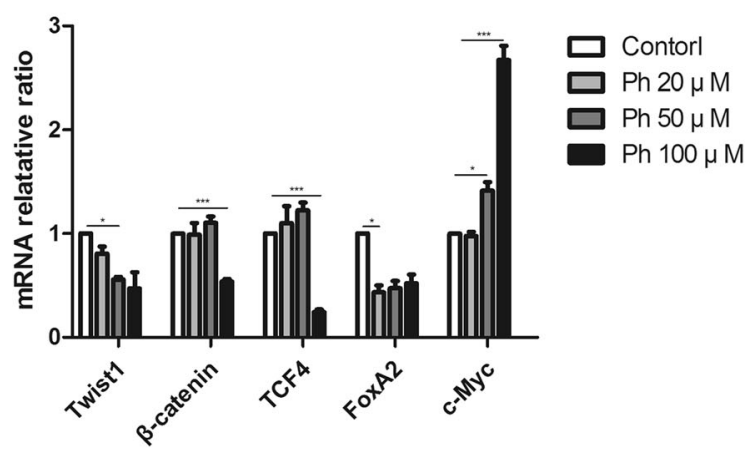

DU145 cells were downregulated. However, c-Myc decreased in PC3 but increased in DU145. Results are presented as means \pm SEM

Publisher's Note Springer Nature remains neutral with regard to jurisdictional claims in published maps and institutional affiliations 